\title{
Prescribing in Pregnancy
}

\section{Psychotropic drugs}

\author{
J B LOUDON
}

At some time during their pregnancy at least $10 \%$ of a random sample of women attending antenatal classes will experience appreciable psychological distress, mainly anxiety. ${ }^{1}$ Of those who have suffered previous psychological illness or undergone termination of pregnancy the figure may be as high as $16 \% .^{2}$ Few women are referred to a psychiatrist during pregnancy. During the puerperium $0 \cdot 2 \%$ of mothers develop a psychotic illness, at least $10 \%$ develop a depressive illness, and a further $16 \%$ suffer a self limiting depressive reaction, qualitatively different from the "birth time blues," which lasts up to a month. ${ }^{3}$ Often these depressive illnesses and reactions are not reported to the patient's general practitioner, but they are corrosive to personal well being and to marital happiness and merit both attention and appropriate treatment.

The distress of pregnancy may be related to adverse events or social circumstances, being more common in unmarried women, and it is probably less amenable to drug treatment. For the disorders of the puerperium, which seem to be caused by a biological disturbance but which occur more often in primigravidas, in the unmarried, in those who have undergone section, and after a perinatal death, drug treatment is likely to be indicated. ${ }^{3 a}$ This article discusses psychotropic drugs, but it should be remembered that other forms of treatment are important and include support from staff or self help groups, psychological treatments such as relaxation exercises or cognitive therapy, and mobilisation of community resources.

Psychotropic drugs used in pregnancy and during the puerperium will be dealt with in four groups: the hypnotic and anxiolytic drugs, neuroleptic drugs, antidepressants, and lithium carbonate.

\section{Safety of psychotropic drugs used in pregnancy and during the puerperium}

\section{HYPNOTIC AND ANXIOLYTIC DRUGS: BENZODIAZEPINES}

Only benzodiazepine drugs should be used; barbiturates and the older anxiolytic drugs such as meprobamate are obsolete. Reports from Scandinavia in the early. 1970s suggested greater use of benzodiazepines by the mothers of children born with defects of the palate, although other studies at the time failed to confirm the finding. Recently, a careful study which compared 611 infants with such deformities and 2498 controls found no evidence of an excess of diazepam use by their mothers. ${ }^{4}$ Some reassurance can therefore be given to an anxious woman who has been taking diazepam in early pregnancy. Abrupt withdrawal of diazepam under these circumstances is not justified.

Royal Edinburgh Hospital, Edinburgh EH10 5HF

J B LOUDON, FRCPSYCH, consultant psychiatrist
There is no evidence to suggest that benzodiazepines have a harmful effect on the fetus later in pregnancy, but the need for these drugs has to be clear to justify their use, especially on a regular basis. Diazepam appears in fetal blood within a few minutes of an intravenous or intramuscular injection, but the fetus seems to have a limited capacity to metabolise the drug. ${ }^{5}$ Pharmacologically, the fetus acts as a "deep compartment," wherein diazepam is slow to accumulate but also, with its active metabolites, is eliminated slowly. This means that regular ingestion of diazepam by the mother will result in accumulation in the fetus. These factors appear to explain the finding that during labour a single bolus of less than $\mathbf{3 0}$ $\mathrm{mg}$ of diazepam has no adverse effect on the infant as measured by the Apgar score. A larger single dose or sustained prenatal benzodiazepine ingestion can lead to the "floppy infant syndrome," which is characterised by hypotonia, respiratory embarrassment, difficulty in suckling, and hypothermia. There is also good evidence for a withdrawal syndrome in infants whose mothers have taken benzodiazepines regularly during pregnancy. ${ }^{6}$

Diazepam is present in maternal blood un-ionised and is lipophilic; it is therefore readily transferred to breast milk. The newborn infant continues to have an impaired ability to metabolise the drug completely, and diazepam has been found in an infant six days after a single dose was given to the mother. Regular use by the mother leads to accumulation in the child. There is little justification for use by nursing mothers of diazepam or any of the long acting, older, drugs such as chlordiazepoxide or nitrazepam. Lorazepam has been associated with neonatal hypotonicity but may not enter breast milk in troublesome amounts. Neonates seem to be able to metabolise oxazepam satisfactorily after the second or third day, ${ }^{7}$ which might justify preference for this drug if a benzodiazepine has to be used by the nursing mother.

\section{NEUROLEPTIC DRUGS: PHENOTHIAZINES AND THIOXANTHINES}

Pregnant women are unlikely to start taking drugs in this group unless as an obsolete and inadvisable treatment for anxiety. A number of women who have suffered from episodes of a functional psychosis may, however, be on long term neuroleptic treatment as prophylaxis and may neglect contraceptive measures as a result of the illness or its after effects. A study of schizophrenic mothers showed that neonatal mortality is twice that of normal controls, ${ }^{8}$ which makes assessment of the dangers of neuroleptic drugs more difficult. Several studies have failed to show a teratogenic effect of neuroleptic drugs, especially trifluoperazine, taken in early pregnancy,' and no lasting behavioural or developmental effects on the child have been found as a result of exposure to neuroleptic drugs in later pregnancy. Prochlorperazine, which is used more often as an antiemetic, has been shown to be teratogenic when the fetus is exposed between the sixth and 10th weeks of gestation. ${ }^{10}$

Neuroleptic drugs enter breast milk in clinically unimportant amounts. There are a few reports of a pseudoparkinsonian reaction in neonates born to mothers taking oral or depot neuroleptic drugs. ${ }^{11}$ 


\section{TRICYCLIC ANTIDEPRESSANTS}

There is a close structural relation between many neuroleptic drugs and the tricyclic antidepressants and much overlap in their pharmacological effects. Not surprisingly, therefore the information about their use during pregnancy and lactation is similar. ${ }^{12}$ The newer non-tricyclic antidepressants are a different matter in that their safety has not yet been proved. Given that two such drugs (zimelidine and nomifensine) have been withdrawn in the past four years because of unexpected toxicity once they came into widespread use, these newer drugs are best avoided in pregnancy.

As happens in adults when tricyclic drugs are suddenly withdrawn, a withdrawal reaction has been reported in some neonates born to mothers who received tricyclic antidepressants in the last month of pregnancy. ${ }^{13}$ The effects include irritability, apparent abdominal cramps, restlessness, insomnia, and fever.

Both neuroleptic drugs and tricyclic antidepressants enter breast milk in detectable quantities, but ingestion of small amounts of these drugs does not appear to affect the neonate. Indeed, follow up studies over long periods of the children of mothers taking chlorpromazine failed to show any adverse effect on development. ${ }^{14}$ There is little published information on the transfer of monoamine oxidase inhibitors to breast milk; tranylcypromine appears to be safe for nursing mothers. ${ }^{\text {is }}$

\section{LITHIUM CARBONATE}

In some parts of the United Kingdom nearly one person in 1000 takes lithium as prophylaxis for recurrent affective illness; a substantial number of these are women of childbearing age. Many studies have confirmed the teratogenic effect of lithium taken in the first months of pregnancy. The cardiovascular system is usually affected, in particular the tricuspid valve (see previous article on identification of fetal abnormality). A recent study has confirmed previous figures of a $7 \%$ risk and given some valuable comparisons. ${ }^{16}$ In a group of pregnant women suffering from manic depression the risk of fetal cardiac abnormalities was increased fivefold in those taking lithium compared with those taking other drugs. Nine of 59 children born to mothers taking lithium were malformed or died soon after birth compared with one of 38 taking drugs other than lithium.

Lithium clearance doubles during pregnancy, which may necessitate an increase in the total daily intake of lithium to maintain the same serum concentration. At the time of delivery the clearance abruptly falls back to normal, and this may be sufficiently fast to precipitate toxic concentrations of lithium in the mother. There is no evidence of a withdrawal reaction or behavioural abnormality in neonates exposed to lithium in utero.

Lithium enters breast milk freely, and serum concentrations in neonates may be close to values that are therapeutic in adults. ${ }^{17} 18$ Toxicity may therefore supervene if there is negative sodium or water balance in the infant.

\section{Guidelines for drug use during pregnancy and in the puerperium}

\section{PATIENTS ALREADY RECEIVING TREATMENT}

The aim of minimising exposure of the fetus and the neonate to psychotropic drugs is helped enormously by patients who are educated and enlightened about treatment. Lines of management differ for those known to have suffered a psychological disorder before the particular pregnancy and those whose illness has arisen for the first time during pregnancy or in the puerperium.

Patients already receiving long term psychotropic treatment for psychosis should take contraceptive meaures or, if no further children are wanted, should be offered sterilisation as the puerperium is a time of high risk of recurrence of a previous psychosis, with all that that implies for the progression of secondary and tertiary handicap. This issue should be discussed with both the patient and her partner, not once but several times, in language which is clear and non-technical to allow a satisfactory decision to be made. If such a patient is determined to conceive it will be a fine clinical decision whether to risk relapse by withdrawing psychotropic drugs or risk the slight possibility of fetal damage. The fetus may be harmed more by the effects of maternal relapse.

Patients receiving long term lithium prophylaxis are a particular problem if the question of another pregnancy is raised. A sudden desire for pregnancy may be a manifestation of a hypomanic mood swing, which is partially controlled with lithium carbonate. Immediate withdrawal of lithium may result in a full blown illness. The issue must be discussed with the couple over several sessions, which will enable the would be mother's mental state to be more fully assessed. If lithium is to be withdrawn it should be done gradually over six or eight weeks, and attempts at conception should be delayed to ensure that no rebound of the illness follows in the weeks after treatment ends. The patient and her partner should be told that the risk of a puerperal psychosis may approach one in five if there was a previous non-puerperal manic depressive episode. After a previous puerperal psychosis the risk is one in five for a manic illness and one in $\mathbf{1 0}$ for a psychotic depressive illness. For previous schizophrenic or atypical psychosis the risk is less (Platz C, Third International Conference of the Marce Society, Nottingham, 1986).

A patient who conceives while taking lithium and who presents early should stop treatment immediately. If further drug treatment is indicated on clinical grounds neuroleptic drugs or tricyclic antidepressants should be prescribed. A pregnancy which has continued for some time with the mother taking lithium before its discovery is not an automatic indication for termination since the fetal cardiac abnormalities caused by this drug can usually be identified by ultrasound (see previous article on identification of fetal abnormality).

There are some patients whose manic depressive illnesses are so severe or difficult to treat or whose adjustment is so precarious that after the first trimester they will need to continue treatment with lithium, or other neuroleptic maintenance treatment, for the rest of the pregnancy. The serum lithium concentration should be maintained at a value as little above $0.5 \mathrm{mmol} / 1$ at 12 hours after ingestion as possible. The daily lithium intake may need to be increased because of the change in the mother's lithium clearance. In these circumstances it is important that contact is established between the doctor monitoring treatment with lithium and other psychotropic drugs and the obstetrician. Obstetric management is easier if the mother is withdrawn gradually from her treatment in the weeks immediately before the estimated date of delivery.

Most puerperal psychoses start soon after delivery, and as withdrawal symptoms do not occur in neonates better protection is conferred by continuing lithium.

A patient still receiving lithium treatment at term should be told to stop taking her tablets as soon as labour starts. No further lithium should be taken during labour and fluid and electrolyte balance must be maintained. The serum lithium concentration must be monitored frequently, and diuretics should be avoided as they will delay lithium excretion.

Ideally, no patient should take benzodiazepines tor more than five or six weeks. Some potential mothers take benzodiazepines regularly, however, and they should be offered help with benzodiazepine withdrawal before conception takes place. Public awareness of the possible effects of drugs on pregnancy and the fetus is increasing, but there is little point in inducing guilt and anxiety, which may put the mother in an impossible predicament. Women who have stopped taking benzodiazepines and who are having to cope with the additional pressures of pregnancy will need extra support or even specific psychological treatment. This could be found in a self help group or given individually by a community psychiatric nurse or health visitor.

Recent work suggests that many patients maintained on standard doses of depot neuroleptic drugs may do well on considerably lower doses and may need less additional treatment during any relapse. ${ }^{19} \mathrm{~A}$ pregnant patient who is receiving depot neuroleptic drugs may therefore have her regular dose reduced within the limitation of her clinical state. Depot neuroleptics take some time to clear after the last dose, which may have to be six or eight weeks before delivery is due so that the neonate is free from neuroleptic drugs. 


\section{Starting treatment in pregnancy}

Treatment should not be skimped just because a patient is pregnant. For anxiety, intermittent use of benzodiazepines is preferable for day or night time exposure to retain the impact of the drug's action, to avoid dependence, and to minimise the effect on the fetus. Benzodiazepines with long half lives and active metabolites such as nitrazepam, diazepam, and chlordiazepoxide are best avoided in favour of drugs such as oxazepam. A depressive illness of the endogenous type, with symptoms such as early morning wakening, diurnal variation, anorexia, poor concentration, guilt, and suicidal ideation, may be treated in the normal way with tricyclic antidepressants if it occurs in pregnancy. If the depression fails to respond to drug treatment electroconvulsive therapy may be used without harming the fetus or causing any long term damage. The need for electroconvulsive therapy seldom occurs, but suicide in the context of a depressive illness in pregnancy is not unknown, and depressive illness at this time demands vigorous action. If treatment is not undertaken problems are likely to occur in the puerperium and afterwards.

\section{Starting treatment in the puerperium}

When a mother has suffered a previous episode of psychosis, whether in the puerperium or at another time, both she and her medical attendants will be concerned about the risk of recurrence. As the risk is about $20 \%$, and the interruption to mothering is so profound and long lasting, it is reasonable to offer such mothers prophylactic drug treatment immediately after delivery. Such a decision should be taken jointly by the general practitioner, obstetrician, and psychiatrist. The woman and her partner need to be fully informed and will want assurances about the effect that treatment will have on her, her ability to look after the child, and her ability to breast feed. If the previous episode was a schizophrenic or depressive one treatment with neuroleptic drugs or tricyclic antidepressants is st:-aightforward and is compatible with breast feeding. Unless there is some guidance on dosage from the treatment of a previous episode, it may be necessary to start with small doses and to work up to chlorpromazine $150 \mathrm{mg}$ a day or its equivalent. For tricyclic antidepressants the dose would be that required in treating an endogenous depressive illness in a young adult-that is, between 100 and $150 \mathrm{mg}$ amitriptyline or its equivalent a day. There is no reason to expect any untoward effects on an infant who is breast fed but the health visitor has an important role in monitoring the child's progress.

In a patient with a history of bipolar manic depressive illness lithium is normally the drug of choice; and breast feeding would be contraindicated. Serum concentrations of lithium should be monitored frequently to allow them to reach a full therapeutic value as soon as possible. Most postpartum psychiatric disorders occur within the first four or six weeks. If the mother seems normal at the end of this period the drug can be gradually withdrawn over the next month or two. If, on the other hand, there have been signs of a modified illness treatment should be continued, using the same criteria for continuing treatment as in episodes not occurring in the puerperium. The development of an illness (albeit modified by prophylactic treatment) should be noted because it will have a bearing on the management of that particular patient after any subsequent pregnancy. Should the drug treatment fail to resolve the symptoms further lines of treatment, including electroconvulsive treatment, should be used on the same basis as at any other time. A mother needs to be in as good a position as possible to care for her child, to maintain her marriage, and to have a satisfactory quality of life; the potential for suicide or infanticide in an untreated or partially treated psychotic mother adds emphasis to this point.

\section{References}

Kumar R, Robson K. Neurotic disturbance during pregnancy and the puerperium. In: Sandler M, Mental illness in pregnancy and the puerperium. Oxford: Oxford University Press, 1979. Cox JL. Psychiatric morbidity and pregnancy: a controlled study of 263 semi rural Ugandan women. Br J Psychiatry 1979;309:1282-5.

$3 \mathrm{Cox} \mathrm{JL}$, Connor Y, Kendell RE. Prospective study of the psychiatric disorders of childbirth. $\mathrm{Br} \mathrm{J}$ Psychiatry 1982;140:111-7.

3a Kendell RE, Chalmers JC, Platz C. The epidemiology of puerperal psychosis. Br $\mathcal{F}$ Psychiar (in press).

4 Rosenberg L, Mitchell AA, Parsella JL, Pashayan H, Louik C, Shapiro S. Lack of relation of oral clefts to diazepam use during pregnancy. $N$ Engl f Med 1983;309:1282-5.

$5 \mathrm{Kanto} \mathrm{JH}$. The use of benzodiazepines during pregnancy, labour and lactation with particular reference to pharmacokinetic considerations. Drugs 1982;23:354-80.

6 Rementeria $\mathrm{JL}$, Bhatt $\mathrm{K}$. Withdrawal symptoms in neonates from intrauterine exposure to diazepam. Pediatr Pharmacol 1977;90:123-6.

7 Tomson G, Lunell NO, Sundwall A, Rane A. Transplacental passage and kinetics in the mother Tomson G, Lunell NO, Sundwall A, Rane A. Transplacental passage and kinetics in the

and newborn of oxazepam given during labour. Clin Pharmacol Ther 1979;25:74-81.
Rieder RD. The offspring of schizophrenics-fetal and neonatal deaths. Arck Gen Psychiatry 1975;32:200-11.

9 Brockington IF, Kumar R. Motherhood and mental illness. London: Academic Press, 1982:249.

10 Edlund MJ, Craig TJ. Antipsychotic drug use and birth defects: an epidemiologic assessment. Compr Psychiatry 1984;25:32-7.

11 Hill RM, Desmond MM, Kay JI. Extrapyramidal dysfunction in an infant of a schizophrenic mother. J Pediatr 1966;69:589-95.

12 Crombie DL, Pinsent RJ, Fleming D. Imipramine in pregnancy. Br Med $\widetilde{J}$ 1972;i:802.

13 Webster PA. Withdrawal symptoms in neonates associated with maternal antidepressant therapy. Lancet 1973;ii:318-9.

14 Kris EB, Carmichael DM. Chlorpromazine maintenance therapy during pregnancy and confinement. Psychiatric Quanterly 1957;31:690-5.

15 Takyi BE. Excretion of drugs in human milk. Am $\mathcal{G}$ Hosp Pharm 1970;28:317-26.

16 Kallen B, Tandberg A. Lithium and pregnancy-a cohort study on manic depressive women. Acto Psychiar Scand 1983;68:134-9.

17 Ananth J. Side effects in the neonate from psychotropic drugs excreted through breast feeding. Am I Psychiatry 1978;135:801-5.

18 Schon M, Amdisen A. Lithium and pregnancy. III. Lithium ingestion by children breast fed by women on lithium treatment. BrMed $\mathcal{F}$ 1973;ii: 138 .

19 Manchanda R, Hirsch S. Low dose maintenance medication for schizophrenia. Br Med $\mathcal{f}$ 1986;293:515-6.

\section{Further reading}

Krauer B, Krauer F, Hytten F. Drug prescribing in pregnancy. Edinburgh: Churchill Livingstone, 1984.

Brockington IF, Kumar R. Motherhood and mental illness. London: Academic Press, 1982.

Kanto JH. Usc of benzodiazepines during pregnancy, labour and lactation with particular reterence to pharmacokinetic considerations. Drugs 1982;23:354-80.

\section{MATERIA NON MEDICA}

\section{Paradises lost}

Nostalgia, a condition happily unknown to the young, may be a harmless indulgence if jointly shared but unrewarding to an outsider. A friend in his seventies, who had commanded a destroyer in the second world war, on a trip to New York with his daughter envisaged hiring a car there and driving to the navy yard at Boston just to show her around. He had taken his ship there from time to time for repairs and maintenance in the war and had often talked of the activities and sense of common purpose he experienced there. I had seen it fairly recently and apart from Old Ironsides-from whose hull British cannon reputedly rebounded-and a museum there was nothing left but a gentle desolation with grass growing on its roads. The visit was not a success, and I should have warned him.

More recently, on a tour, a fellow traveller described his first visit to Petra, immortalised as the "Rose-red city-chalf as old as Time!" Not all that long ago one had to camp in a rest house, tent, or adjacent cave before descending the gorge. There is now an excellent small single storey hotel discreetly tucked out of sight at the top of the gorge, so it is all very easy, allowing an early morning start down with few people about. I found it quite as marvellous as he described, but the rest of his tale was strangely similar to the earlier episode. Impressed as he had been, he invited a friend to join him on a trip, but the result was disaster. There was the usual typical argument whether to go down the gorge before or after lunch, and, the latter chosen, they were overwhelmed by coachloads of liner passengers, who can now make the return day trip up from the post of Aqaba. The result was pandemonium with many having to make the way up and down on foot; indeed, in the not impossibly distant future I can visualise a helipad at the top-and, for that matter, at the bottom-of the gorge as well.

So I never enthuse to others over places I have seen in the past, and when perchance I have visited them again 30 or $\mathbf{4 0}$ years later I have just been content to feel I had survived so long and so easily with memory intact of bygone days.-ROBERT CUTLER, Surbiton, Surrey. 